\title{
Efeitos da Terapia com Raloxifeno na Pós-menopausa sobre a Espessura Endometrial, Volume Uterino e Perfusão nas Artérias Uterinas
}

Autor: George Dantas de Azevedo

Orientador: Prof. Dr. Marcos Felipe Silva de Sá

Dissertação de Mestrado apresentada ao Departamento de Ginecologia e Obstetrícia na Faculdade de Medicina de Ribeirão Preto da Universidade de São Paulo, em 18 de dezembro de 2000.

Objetivos: determinar os efeitos da terapia com raloxifeno sobre o útero de mulheres na pós-menopausa, por meio da ultra-sonografia transvaginal com metodologia Doppler.

Pacientes e Métodos: foram estudadas, de forma prospectiva, vinte e cinco mulheres na pós-menopausa, sem patologias associadas, atendidas no Departamento de Ginecologia e Obstetrícia da Faculdade de Medicina de Ribeirão Preto. As pacientes foram tratadas com cloridrato de raloxifeno na dosagem de $60 \mathrm{mg}$ ao dia, durante o período de 6 meses. Todas foram submetidas a exame ultra-sonográfico transvaginal com Doppler (equipamento ATL-HDI 3000), antes do início do tratamento e após 30, 90 e 180 dias. Utilizando a metodologia Doppler, foram determinados os indices de resistência (IR) e pulsatilidade (IP) das artérias uterinas direita e esquerda, sendo considerados como indicadores da perfusão uterina. As variáveis analisadas foram: espessura endometrial, volume uterino e os índices de resistência e pulsatilidade das artérias uterinas. A análise estatística foi realizada utilizando a análise de variância para medidas repetidas.

Resultados: no período pré-tratamento, a espessura endometrial foi de 3,38 $\pm 0,73 \mathrm{~mm}$, sendo observados valores semelhantes após 30, 90 e 180 dias de uso do raloxifeno $(3,04 \pm 0,82 ; 3,3 \pm 0,83 ; 3,37 \pm 0,79$; respectivamente) $(p>0,05)$. Não foram observadas diferenças estatisticamente significativas entre os valores obtidos para o volume uterino, nos periodos pré e póstratamento. Os parâmetros de perfusão nas artérias uterinas, avaliados com o método Doppler (IR e IP), também não apresentaram variações significativas ( $p>0,05)$, sendo mantido um padrão de fluxo de alta impedância durante todo o periodo de tratamento com raloxifeno.

Conclusões: no grupo estudado, a terapia com raloxifeno na dosagem de $60 \mathrm{mg}$ ao dia, durante um período de 6 meses, não promoveu aumento da espessura endometrial de mulheres na pós-menopausa. O volume uterino e a perfusão nas artérias uterinas também não apresentaram alterações significativas durante o período de tratamento. Os resultados sugerem que, a curto prazo, a terapia com raloxifeno não afeta o útero de mulheres na pós-menopausa.

Palavras-chave: Menopausa. Climatério. Modulador seletivo do receptor estrogênico.

\section{Associação entre a Incisura Bilateral das Artérias Uterinas Maternas ao Exame pelo Doppler e a Histologia do Leito Placentário}

Autora: Regina Amélia Lopes Pessoa de Aguiar

Orientador: Prof. Dr. Antônio Carlos Vieira Cabral

Tese de Doutorado apresentada ao Programa de Pós-graduação em Ginecologia e Obstetrícia da Faculdade de Medicina da Universidade Federal de Minas Gerais - Área de Concentração em Perinatologia, em 18 de agosto de 2000.

Com o objetivo de avaliar a associação entre a presença da incisura diastólica nas artérias uterinas maternas e as alterações histopatológicas dos vasos úteroplacentários, realizamos estudo transversal incluindo 144 pacientes com gestação única interrompidas por cesariana entre 27 e 41 semanas. Destas 84 gestações estavam associadas à pré-eclâmpsia e 60 não apresentaram intercorrências clínicas. A dopplerfluxometria das artérias uterinas foi sempre realizada pelo mesmo examinador e com um intervalo máximo de 7 dias da interrupção da gestação. Consi- deramos como alterada a presença da incisura diastólica bilateral. As biópsias de leitos placentários foram obtidas por via abdominal. Dos fragmentos obtidos por biópsia $61 \%$ foram considerados à histologia como representativos de leito placentário. A associação entre incisura diastólica bilateral das artérias uterinas e alterações fisiológicas no leito placentário mostrou-se significativa ( $p=0,0000)$. Encontramos uma sensibilidade de $70 \%$, especificidade de $90 \%$ e valores preditivos positivo e negativo de 44 e $97 \%$, respectivamente. A associação entre incisura diastólica bilate- 
ral das artérias uterinas e arteriopatia decidual também foi significativa ( $\mathrm{p}=0,0000)$. A sensibilidade foi de $96 \%$, especificidade de $70 \%$ e valores preditivos positivo e negativo de $26 \%$ e $99 \%$, respectivamente. Em relação à arterioloesclerose encontramos sensibilidade de $80 \%$, especificidade de $55 \%$ e valores preditivos positivo e negativo de $17 \%$ e $96 \%$, respectivamente $(\mathrm{p}=$ $0,0470)$. Concluímos que a incisura diastólica nas artérias uterinas maternas é indicador seguro de vasculopatia no leito placentário. A adequada invasão trofoblástica do leito placentário, revelada por histologia típica de alterações fisiológicas, resulta na ausência de incisura diastólica bilateral das artérias uterinas maternas.

Palavras-chave: Placenta. Dopplervelocimetria. Gravidez normal. Hipertensão arterial.

RBCO 23(4):262,2001

Resumo de Tese

\section{Avaliação do Efeito da Tíbolona, Sobre a Resistência Arterial, em Mulheres na Pós-menopausa}

Autora: Mônica Leite Grinbaum

Orientador: Prof. Dr. José Arnaldo de Souza Ferreira

Tese apresentada ao curso de pós-graduação da Faculdade de Ciências Médicas da Santa Casa de São Paulo em 13 de dezembro de 2000, para obtenção do título de mestre em tocoginecologia.

O objetivo deste estudo foi avaliar o efeito da tibolona sobre a resistência arterial periférica, através da determinação do índice de pulsatilidade (IP) das artérias carótidas comum, radial e poplitea, em mulheres na pós-menopausa. Foram estudadas 29 pacientes num estudo longitudinal, prospectivo, tipo "antes e depois", com sete meses de duração. As pacientes não apresentavam doenças cardiovasculares, quaisquer condições que pudessem interferir na resistência vascular ou qualquer contra-indicação para TRH (terapia de reposição hormonal). Foram administrados $2,5 \mathrm{mg}$ de tibolona, via oral, de forma contínua, por um período de seis meses. Antes, após três e seis meses do início da administração e após um mês da interrupção da droga foram realizadas Doppler ultra-sonografias dúplex a cores das artérias carótida comum, radial e poplítea, direitas e esquerdas para determinação do IP, os quais foram calculados através das médias dos valores en- contrados nos dois lados. Os valores pré-tratamento serviram como controles. Os resultados obtidos foram analisados estatisticamente pela ANOVA. Na artéria carótida comum não foram observadas diferenças significantes do IP no terceiro e sexto mês, em relação ao pré-tratamento. $\mathrm{Na}$ artéria radial foi observada redução siginificante do IP no sexto mês em relação ao pré-tratamento (queda de $55 \%$ ). Na artéria poplítea o IP também sofreu redução significante no sexto mês em relação ao pré-tratamento (queda de 30\%). Após a análise final dos resultados, foi possivel concluir que não houve variação do IP da artéria carótida comum; mas, sim, na radial e na poplitea, artérias cujas resistências sofreram redução após seis meses do início da terapêutica com tibolona.

Palavras-chave: Terapia de reposição hormonal. Menopausa. Dopplervelocimetria.

RBCO 23(4):262-263,2001

Resumo de Tese

\section{Repercussões da Terapia de Reposição Hormonal em Ratas Hipertensas e Ooforectomizadas}

Autor: Rogério Dias

Tese apresentada à Faculdade de Medicina de Botucatu - UNESP- para obtenção de livre-docente, em 13 março de 2001.

Objetivo: avaliar o efeito da estrogenioterapia sobre a castração cirúrgica, comparando-a com a "menopausa espontânea"; a evolução dos niveis plasmáticos de colesterol total, HDL-colesterol, LDL-colesterol, relação LDL/HDL-colesterol, triglicérides, sódio, potássio, uréia e creatinina; a evolução da pressão arterial; e o peso corpóreo de ratas hipertensas e ooforectomizadas. Material e Métodos: foram utilizadas 60 ratas virgens, da linhagem "Wistar", pesando entre 260 e 300 gramas e com idade entre 5 e 6 meses. As ratas foram sorteadas para compor os 5 grupos experimentais (G1-C controle - "menopausa espontânea"; G2-O, ooforectomizada; G3-OH, ooforectomizada + hipertensão, G4-OE, ooforectomizada + estrogenioterapia e G5-OHE, ooforectomizada + hipertensão + estrogenioterapia). A hipertensão foi induzida, experimentalmente, pela téc- 
nica de Goldblatt II ( 2 rins - 1 clipe), que consiste na contrição da artéria renal esquerda. Posteriormente, foram realizadas medidas periódicas da pressão arterial pelo método de pletismografia de cauda. A ooforectomia bilateral foi realizada para induzir as ratas a um estado de privação estrogênica. $O$ experimento foi analisado, distintamente, em 4 momentos para a pressão arterial e peso corpóreo, e em 3 momentos para as dosagens bioquímicas.

Resultados: as ratas dos grupos G3-OH e G5-OHE apresentaram elevação dos níveis de pressão arterial acima do normal; a ooforectomia bilateral (grupos G2-O e G4-OE) não determinou alteração de peso corpóreo, enquanto as variáveis bioquimicas sofreram alterações com a hipertensão e com a ooforectomia, pois voltaram a valores normais após a suplementação estrogênica.

Conclusões: o modelo experimental foi adequado para o objetivo de nosso estudo, pois permitiu obtenção de animais hipertensos. A castração cirúrgica, na ausência de reposição hormonal, alterou precocemente o metabolismo lipídico das ratas em relação às "menopausadas espontaneamente". A estrogenioterapia normalizou os niveis plasmáticos do colesterol total, HDL-colesterol, LDL-colesterol, da relação LDL/ HDL-colesterol, porém aumentou os niveis de triglicérides das ratas hipertensas e ooforectomizadas. A estrogenioterapia abaixou os niveis de pressão arterial, não interferiu no peso corpóreo e diminuiu os níveis plasmáticos de sódio, potássio, uréia e creatinina das ratas hipertensas e ooforectomizadas.

Palavras-chave: Terapia de reposição hormonal. Ooforectomia. Hipertensão arterial. Modelos experimentais.

Estudo do Índice do Liquido Amniótico em Gestantes com Hipertensão Arterial e sua Relação com a Vitalidade Fetal e Resultados Perinatais

Autor: Alexandre Provinciatto

Orientadores: Prof. Dr. Antonio Fernandes Moron e Prof. Dr. Luiz Kulay Junior

Tese apresentada à Universidade Federal de São Paulo - Escola Paulista de Medicina, para obtenção do título de Doutor em Medicina, em 25 de setembro de 2000.

No decorrer da gravidez, o volume do líquido amniótico (VLA) varia amplamente: com o estado normal ou patológico, com a idade da gestação e de paciente para paciente em um mesmo período. Em decorrência destes aspectos, impõem-se estudos para a avaliação do VLA, quer em gestações consideradas normais ou patológicas. Consideradas as gestações de alto risco, o índice do líquido amniótico (ILA), quando avaliado como um adjunto à investigação fetal anteparto, tem mostrado relacionar-se bem com a vitalidade fetal e resultados perinatais. A ultra-sonografia seriada, principalmente no que se refere à avaliação do VLA, é considerada imprescindivel na avaliação da vitalidade fetal em gestantes com risco para oligoidrâmnio, particularmente com hipertensão arterial, contribuindo para a profilaxia da morbimortalidade perinatal. O objetivo deste trabalho foi estudar o ILA através da técnica ultrasonográfica dos quatro quadrantes em gestantes com hipertensão arterial e sua relação com a vitalidade fetal e resultados perinatais. Realizamos estudo prospectivo em cento e cinqüenta e uma pacientes. Os critérios de exclusão foram: (1) doenças maternas outras responsabilizadas por alterações no liquido amniótico e pressão arterial; (2) exposição a drogas; (3) infecções pré-natais; (4) anomalias estruturais detectadas no feto ou no recém-nascido; (5) rotura prematura das membranas; (6) gemelidade; (7) perda de seguimento, ou seja, parto em outro serviço. As gestantes foram divi- didas em três grupos, de acordo com o resultado do ILA: grupo 1, abaixo do percentil 2,5 (oligoidrâmnio); grupo 2, entre os percentis 2,5 e 5 (ILA diminuído); grupo 3, entre os percentis 5 e 95 (normal). Foram aplicados os testes do qui-quadrado $\left(\chi^{2}\right)$ para tabelas de contingência, com a finalidade de comparar os três grupos em relação às freqüências com que ocorreram as diversas características estudadas. Houve associação entre oligoidrâmnio e as alterações nos exames de avaliação da vitalidade fetal: cardiotocografia anteparto ou intraparto e dopplervelocimetria da artéria umbilical. Encontrou-se relação entre oligoidrâmnio e os seguintes resultados perinatais adversos: pré-termo; peso ao nascimento inferior a $2.500 \mathrm{~g}$; recém-nascidos pequenos para a idade gestacional; índice de Apgar menor que sete no primeiro minuto; morbidade perinatal; tempo de permanência no berçário maior ou igual a sete dias; líquido amniótico meconial; parto cesáreo; parto cesárea por sofrimento fetal e sofrimento fetal anteparto ou intraparto. Os resultados demonstram que os fetos de gestantes hipertensas com associação de oligoidrâmnio são potencialmente suscetíveis à hipóxia, principalmente durante o período intraparto e tendem a apresentar sinais de asfixia após o nascimento.

Palavras-chave: Índice do líquido amniótico. Hipertensão arterial. Vitalidade Fetal. Resultados perinatais. 


\title{
Avaliação do Uso da Metformina em Pacientes Portadoras da Sindrome dos Ovários Policísticos.
}

Autor: Sandro Magnavita Sabino

Orientador: Prof. Dr. Selmo Geber

Dissertação de Mestrado apresentada ao Curso de Pós-Graduação em Ginecologia e Obstetrícia da Faculdade de Medicina da Universidade Federal de Minas Gerais, em 13 de dezembro de 2000.

O objetivo deste estudo clínico foi avaliar o uso da metformina como opção terapêutica para pacientes portadoras da sindrome dos ovários policísticos (SOP). Foi realizado um estudo randomizado duplo cego, com a utilização de placebo. Vinte e duas pacientes foram divididas em dois grupos, ambos com 11 pacientes. $\mathrm{O}$ grupo A utilizou placebo, por um periodo de três meses e o grupo B utilizou a metformina $850 \mathrm{mg}$, de 12/ 12 horas, pelo mesmo periodo de tempo. As avaliações laboratoriais, clínicas e ultra-sonográficas foram realizadas antes do início do tratamento e após os três meses. Todas as pacientes apresentavam alterações do ciclo menstrual; $54,5 \%$ eram obesas; $41 \%$ eram hirsutas e $72,7 \%$ apresentavam hiperandrogenismo. A resistência insulínica foi encontrada em $45,5 \%$ dos casos; $72,7 \%$ apresentavam história familiar positiva para a sindrome e $86,3 \%$ das pacientes apresentavam ovários com características polimicrocísticas ao exa- me de ultra-som. Os grupos A e B foram considerados comparáveis. Após os três meses de tratamento $90 \%$ das pacientes que utilizaram a metformina (Grupo B) apresentaram regularização do ciclo menstrual, houve redução dos niveis de insulina e da resistência insulínica. Os niveis de testosterona livre e colesterol também sofreram redução, porém sem significância estatística. Neste grupo B, 36\% relataram queixas leves referentes ao trato gastrintestinal que melhoraram após a primeira semana de tratamento. Concluímos que a metformina é uma boa opção para o tratamento de pacientes portadoras da SOP, devido a sua eficácia e boa tolerabilidade.

Palavras-chave: Síndrome dos ovários policísticos. Resistência à insulina. Hiperandrogenismo. Distúrbios menstruais.

\section{XIJORNADAAMAZONENSEDE GINEOOLOGIAEOBSTETRÍCIA}

\section{3 a 04 de Agosto de 2001}

\author{
Manaus-AM
}

\section{Organização: $S A G O$}

\section{Tel.: (0xx92) 232-2401}

